\title{
EFEITOS DA SUPLEMENTACCÃO DE FIBRAS SOLÚVEIS, DIETA DE CAFETERIA E EXERCÍCIO FÍSICO SOBRE AORTA TORÁCICA DE RATOS
}

\section{Patricia Batista Travassos}

Doutoranda do Programa de Pós-Graduação em Ciências Biológicas da Universidade Estadual de Maringá (UEM), Maringá (PR), Brasil.

E-mail: patriciatravassos2014@gmail.com

\section{João Victor Del Conti Esteves}

Doutorando do Programa de Pós-Graduação em Fisiologia Humana do Instituto de Ciências Biomédicas da Universidade de São Paulo (USP), SP, Brasil.

\section{Solange Marta Franzoi de Moraes}

Departamento de Ciências Fisilógicas da Universidade Estadual de Maringá (UEM), Maringá (PR), Brasil.

\section{Célia Regina de Godoy Gomes}

Departamento de Ciências Morfológicas da Universidade Estadual de Maringá (UEM), Maringá (PR), Brasil.
RESUMO: As fibras alimentares vêm despertando interesse de especialistas dasáreas de nutrição e saúde em geral. Elas contribuem para a redução do risco de desenvolvimento de doenças cardiovasculares, além de ajudar no processo de perda de peso, principalmente quando seu consumo é associado à atividade física. Entretanto, existem poucos estudos associando o uso de fibras solúveis à uma dieta hipercalórica e ao exercício físico. Assim, o objetivo desse estudo foi investigar os efeitos das fibras solúveis sobre a aorta torácica e perfil lipídico de ratos machos wistar sedentários e treinados submetidos à dieta de cafeteria. Os parâmetros morfométrico e estereológico foram: espessura íntimamédia e densidade de volume do músculo liso e do colágeno e o perfil lipídico foi, então, determinado. Não foram observadas alterações na espessura da parede vascular, densidade de volume do músculo liso e colágeno. No entanto, o perfil lipídico apresentou-se alterado com o uso da dieta de cafeteria. A fibra alimentar associada ao treinamento aeróbio e a uma dieta balanceada apresentou resultado eficaz, não alterando a morfologia do vaso.

PALAVRAS-CHAVE: Aorta; Dieta; Fibras na Dieta; Obesidade.

\section{EFFECTS OF SUPPLEMENTATION BY SOLUBLE FIBERS, CAFETERIA DIET AND PHYSICAL EXERCISE ON RATS' THORACIC AORTA}

\begin{abstract}
Food fibers have recently been in the limelight of nutrition and health experts. They reduce the development of cardiovascular diseases and help in the process of loss weight especially when their intake is associated with physical activity. However, few studies are extant on the use of soluble fibers associated to a hypercalorie diet and physical exercise. Current analysis deals with the effects of soluble fibers on the thoracic aorta and lipid profile of sedentary and trained male Wistar rats subjected to a cafeteria diet. Morphometric and stereological parameters comprised mid thickness and density of the volume of the smooth muscle and collagen, and lipid profile. No changes were reported on the thickness of the vascular wall, density of volume of the smooth muscle and collagen. However, lipid profile was modified due to the cafeteria diet. Food fiber associated with aerobic training and with a balanced diet provided an efficacious result with no alteration in the vase's morphology.
\end{abstract}

KEY WORDS: Aorta; Diet; Fibers in the Diet; Obesity. 


\section{INTRODUÇÃO}

Influenciadas pelos avanços tecnológicos na indústria de alimentos e pela globalização da economia, as práticas alimentares contemporâneas têm sido objeto de preocupação das ciências da saúde, desde que os estudos epidemiológicos passaram a sinalizar estreita relação entre a dieta e doenças crônicas associadas à alimentação, dentre elas a obesidade, sendo esta caracterizada pelo acúmulo excessivo de gordura que passa a comprometer a saúde do indivíduo, pois está associada ao aumento dos riscos para o acometimento de doenças cardíacas, aterosclerose, hipertensão arterial, hiperlipidemias e diabetes (COUTINHO; GENTIL; TORAL, 2008; MONTEIRO; RIETHER; BURINI, 2004; PROENÇA, 2010; GARCIA, 2003).

As fibras alimentares são definidas como a porção de hidrato de carbono comestiveis das plantas, resistentes à digestão enzimática, são constituídas por fibras solúveis (pectinas) e insolúveis (lignina). Como um nutriente essencial, têm apresentado um papel crucial na manutenção da saúde, com ações de prevenção e proteção para várias doenças humanas, tais como diabetes, doenças cardiovasculares e outras (MATTOS; MARTINS, 2000; BERNAUD; RODRIGUES, 2013; SLAVIN, 2013; ZHANG et al., 2016).

O exercício físico regular atua na prevenção e controle das doenças cardiovasculares, principalmente quando associado a modificações na alimentação (MANDICA et al., 2010; PINHO et al., 2010; RIQUE; SOARES; MEIRELLES, 2002). A combinação do exercício físico e do uso de fibras alimentares pode ser crucial para a manutenção do peso corporal e prevenção de doenças e dislipidemias. Portanto o presente estudo visou investigar o efeito da suplementação com fibras solúveis e do treinamento físico sobre parâmetros morfométrico e estereológico da aorta torácica e perfil lipídico de ratos submetidos a uma dieta de cafeteria.

\section{METODOLOGIA}

\subsection{MATERIAL}

Utilizou-se 20 ratos da linhagem Wistar, com idade de 60 dias. Durante o período experimental os animais foram mantidos em biotério com fotoperíodo controlado automaticamente (ciclo claro/escuro $12 \mathrm{~h} / 12 \mathrm{~h}$ ) e sob temperatura de $22 \pm 1{ }^{\circ} \mathrm{C}$. Os animais foram divididos aleatoriamente em 4 grupos, de acordo com as condições de dieta alimentar, ingestão de fibras e treinamento físico: Treinado Controle Fibras (TCF), Treinado Cafeteria Fibras (TCaF), Sedentário Controle Fibras (SCF) e Sedentário Cafeteria Fibras (SCaF). Todos os animais envolvidos na pesquisa foram pesados 2 vezes por semana. Os procedimentos experimentais que envolveram o uso de animais foram aprovados pelo Comitê de Ética em Experimentação Animal (Parecer n ${ }^{\circ}$ 048/2006).

\subsubsection{Dieta Alimentar}

Os animais dos grupos controle (SCF e TCF) receberam água ad libitum e uma dieta alimentar balanceada (ração Nuvilab ${ }^{\circledR}$ ), que é composta de carbonato de cálcio, farelo de milho, farelo de soja e de trigo, fosfato bicálcio, cloreto de sódio, multivitamínico e aminoácidos.

Os animais submetidos à dieta de cafeteria (SCaF e TCaF) consumiram: água, salsicha, chips, pão francês, mortadela, paçoca, refrigerante, ração e marshmallow, ingeridos nos diferentes dias da semana, sendo esses alimentos pesados e separados todos os dias.

Foi oferecido a estes animais acesso à mesma ração comercial oferecida aos animais controle, visando avaliar as preferências dos animais por uma dieta mais balanceada e menos palatável (ração comercial) ou por uma dieta nutricionalmente menos equilibrada e mais palatável (dieta de cafeteria).

A dieta foi disponibilizada e monitorada diariamente. Os alimentos foram oferecidos sem nenhuma modificação, sendo disponibilizados na mesma condição em que eram removidos da embalagem. A quantidade oferecida de cada alimento foi dimensionada sempre visando exceder seu consumo.

\subsubsection{Ingestão de Fibras}

As fibras alimentares solúveis (Resourse FiberMais - Nestlé $e^{\circledR}$ ) foram dissolvidas em água e 
administradas via gavagem $(0,5 \mathrm{ml})$. Os quatro grupos de animais (TCF, TCaF, SCF e SCaF) receberam inicialmente $10 \mathrm{mg} /$ dia de fibras, com aumentos crescentes (10 mg a cada duas semanas) até que chegasse a $30 \mathrm{mg} /$ dia/animal, adaptando as recomendaçóes de uso para adultos do fabricante.

\subsubsection{Treinamento Físico}

Os animais dos grupos TCF e TCaF, a partir da $5^{\mathrm{a}}$ semana do início da dieta de cafeteria, iniciaram o treinamento físico aeróbico. O protocolo de treinamento ocorreu 5 vezes/semana, durante 8 semanas. O exercício físico foi realizado em esteira ergométrica programável

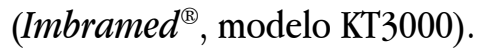

Os animais foram submetidos inicialmente a uma semana de adaptação (10 minutos/dia, à velocidade de $0,3 \mathrm{~km} / \mathrm{h}$ a $0,6 \mathrm{~km} / \mathrm{h}$, em estágios de 2 minutos cada), em seguida, a velocidade e o tempo foram gradativamente aumentados até se atingir a uma hora/ dia, em intensidade moderada (60 a 65\% do $\mathrm{VO}_{2}$ máx.), de acordo com protocolo estabelecido por Dufloth et al. (1997), adaptado de Negrão et al. (1992).

\subsection{MÉTODOS}

Após 12 semanas de período experimental os animais foram anestesiados com injeção endovenosa de Pentobarbital sódico ( $4 \mathrm{mg} / 100 \mathrm{~kg}$ ). Em seguida, foram submetidos à laparotomia mediana para a coleta de sangue ( $4 \mathrm{~mL}$ ) da veia cava inferior e realizada a dissecação para retirada da aorta torácica, onde foi utilizado a parte descendente, retirada a partir do ápice do arco da aorta (ponto mais cranial da aorta). As amostras sanguíneas foram centrifugadas (3000 rpm, 10 minutos) e as análises foram feitas a partir do sobrenadante.

\subsubsection{Dosagens Bioquímicas}

A determinação das concentrações plasmáticas de colesterol total, colesterol de alta (HDL-C) e baixa (LDL-C) densidades e triglicerídeos foi realizada utilizando kits de métodos enzimáticos colorimétricos em espectrofotômetro.

\subsubsection{Análise Histológica}

As aortas torácicas após serem retiradas foram fixadas em formol tamponado a $10 \%$. As peças foram desidratadas em etanol e diafanizadas em xilol. As peças foram incluídas em parafina, em seguida foram realizados cortes histológicos transversais com $6 \mu \mathrm{m}$ de espessura no aparelho Micrótomo (Leica RM 2145), e desparafinados por xilol e hidratadas em série decrescente por álcool.

Os cortes foram corados pelos seguintes métodos: Tricrômico de Masson e Orceína-picrosíriushematoxilina. A orceína utilizada foi a TAENZER-UNA com modificação de ROMAIS (BEHMER et al., 1976).

Para a morfometria da parede da aorta torácica foi feita a medida íntima-média, onde foram analisadas quatro regiões diferentes de um mesmo corte diametralmente opostas para maior precisão do estudo. Foi estudada a densidade de volume (Vv) do colágeno e do músculo liso na túnica média da aorta torácica. Para cada animal, foram estudados 15 cortes histológicos em um campo microscópico aleatório. A contagem de pontos foi realizada através do sistema-teste de 36 pontos total.

A análise microscópica foi realizada em sistema de vídeo-microscópio (Microscópio Olympus Bx 50 acoplado a um sistema de captura de imagem - software Image Pro Plus) através de objetivas de 20 e 40x.

A densidade de volume ( $\mathrm{Vv}$ ) foi calculada pela seguinte fórmula:

$$
\mathrm{Vv}=\frac{\mathrm{Pp}}{\mathrm{Pt}} \underline{\%}
$$

Onde:

- Pp é o número de pontos que tocam a estrutura em estudo;

- Pt é o número total de pontos do sistema teste e igual a 16.

\subsection{ESTATÍSTICA}

Avaliou-se os resultados apresentados como média \pm erro padrão (SEM) por análise de variância (ANOVA) One-way seguido por teste de Tukey utilizandose o programa GraphPad Prism - versão 5.0 com nível de significância de $5 \%(p<0,05)$. 


\section{RESULTADOS}

A evolução do ganho de massa corporal dos animais do início ao final do experimento está apresentada na Figura 1.

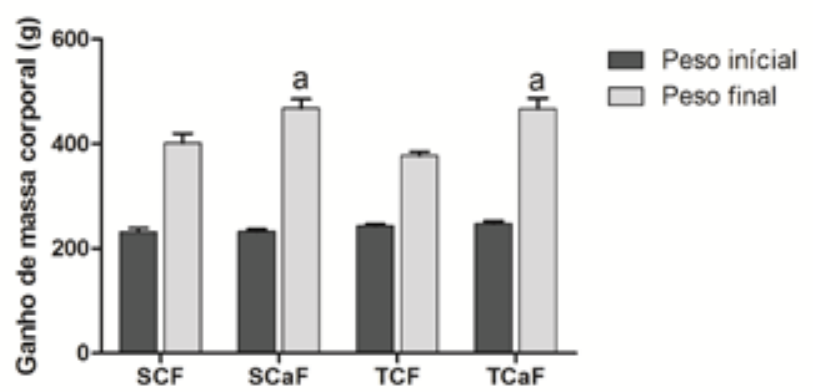

Figura 1. Ganho de massa corporal dos animais no início e final do experimento. Os valores obtidos foram expressos com média \pm erro padrão de $4-5$ animais. (a) $p<0,05$ comparado ao TCF
Os resultados de colesterol total, triglicerídeos, HDL, LDL estão apresentados na Figura 2. O colesterol total não apresentou diferença significativa entre os grupos (Figura 2A), apesar do grupo SCaF apresentar uma tendência de valores menores de colesterol no sangue. Já os triglicerídeos (Figura 2B) apresentaram diferenças significativas nos grupos cafeteria em relação aos grupos controles $(p<0,05)$ e nos grupos treinados em relação ao sedentário $(p<0,05)$. O colesterol HDL (Figura 2C) apresentou diferença no grupo $\mathrm{SCaF}$ em relação aos grupos SCF e TCF $(p<0,05)$, indicando que o exercício físico com a suplementação de fibras e não associado a uma dieta calórica teve uma influência positiva no HDL.

$O$ índice do LDL não foi estatisticamente significativo, como pode ser observado na Figura 2D.
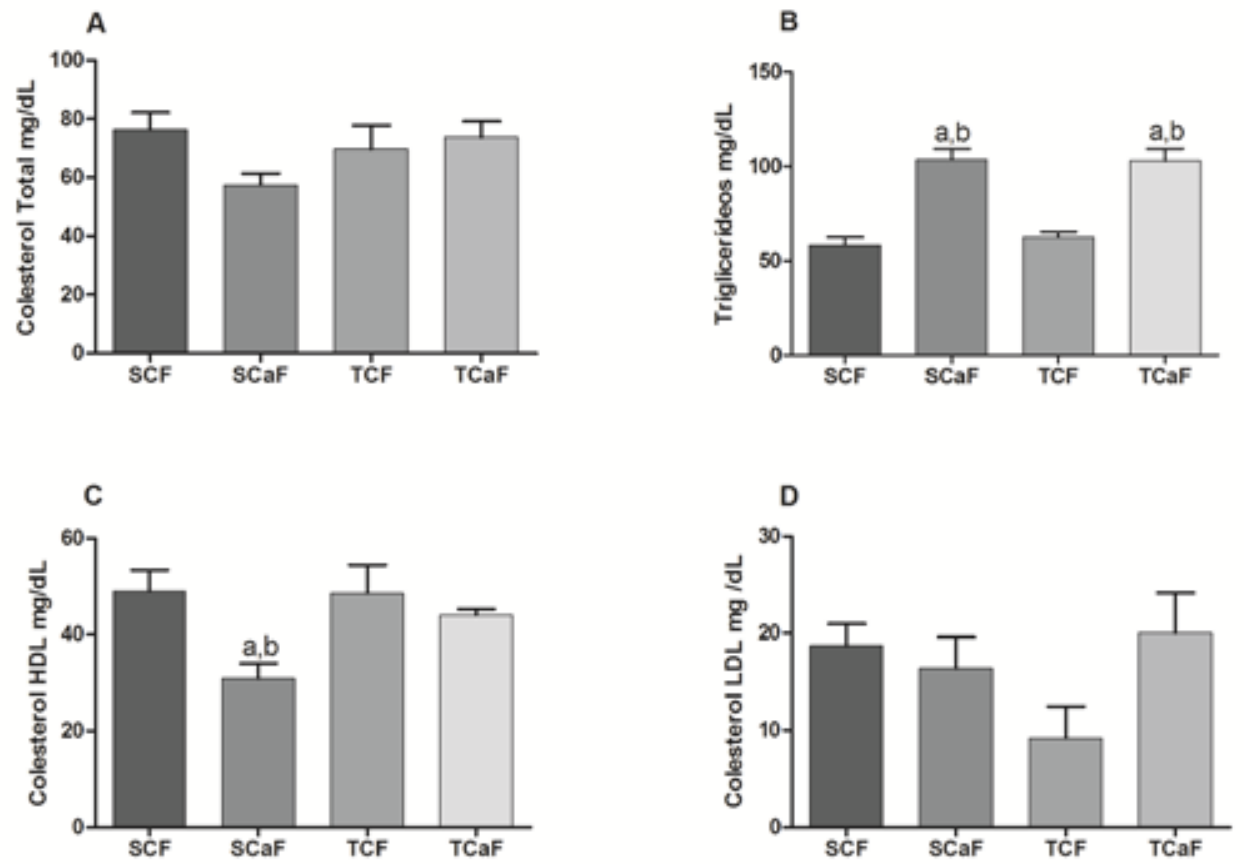

Figura 2. Concentração de colesterol total, Triglicerídeos, HDL, LDL no sangue de ratos submetidos à dieta de cafeteria, treinamento crônico e suplementação com fibras solúveis. Os valores obtidos foram expressos com média \pm erro padrão de $4-5$ animais. (a) $p<0,05$ comparado ao TCF (b) $p<0,05$ comparado SCF

A análise ao microscópio de luz em secções transversas da aorta torácica não revelou alterações significativas (Figuras 3 e 4). A túnica média apresentouse com lâminas elásticas dispostas em camadas alternadas com células musculares lisas. As células musculares lisas da túnica média estavam arranjadas obliquamente no plano de secção e as fibras colágenas e fibrilas elásticas estavam entrelaçadas a elas. A Figura 3B mostra maior espessura da parede do vaso no grupo SCaF em relação aos demais grupos, porém, na análise estatística da morfometria íntima-média não foi observada diferença significativa entre os grupos (Tabela 1).

Nos quatro grupos em estudo observouse espessamento da túnica íntima e fragmentação e duplicação da limitante elástica interna (Figuras 3 e 4). 
O componente elástico na túnica média (Figura 4) estava composto por 7-12 lâminas concêntricas orientadas em direção paralela e arranjadas cilindricamente ao redor da luz do vaso, presentes desde a íntima até a adventícia, e unidas por lamínulas elásticas.

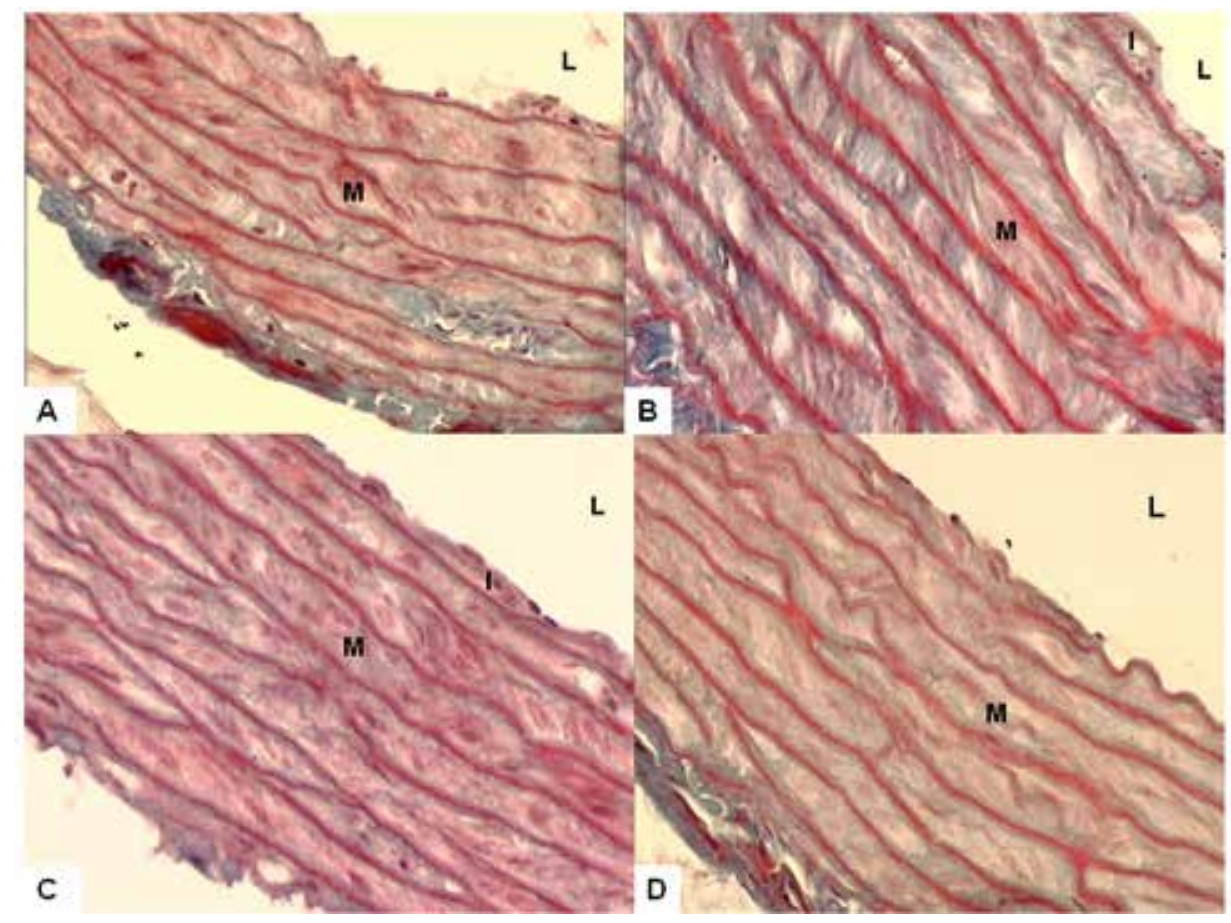

Figura 3. Fotomicrografia da aorta torácica dos grupos SCF (A), SCaF (B), TCF (C) e TCaF (D). Em A, B, C e D a túnica média (M) exibe estrutura normal, com músculo liso disposto entre lâminas elásticas e o colágeno ao redor do músculo liso. $\mathrm{I}=$ espessamento da íntima; $\mathrm{L}=$ luz. Masson, 640x

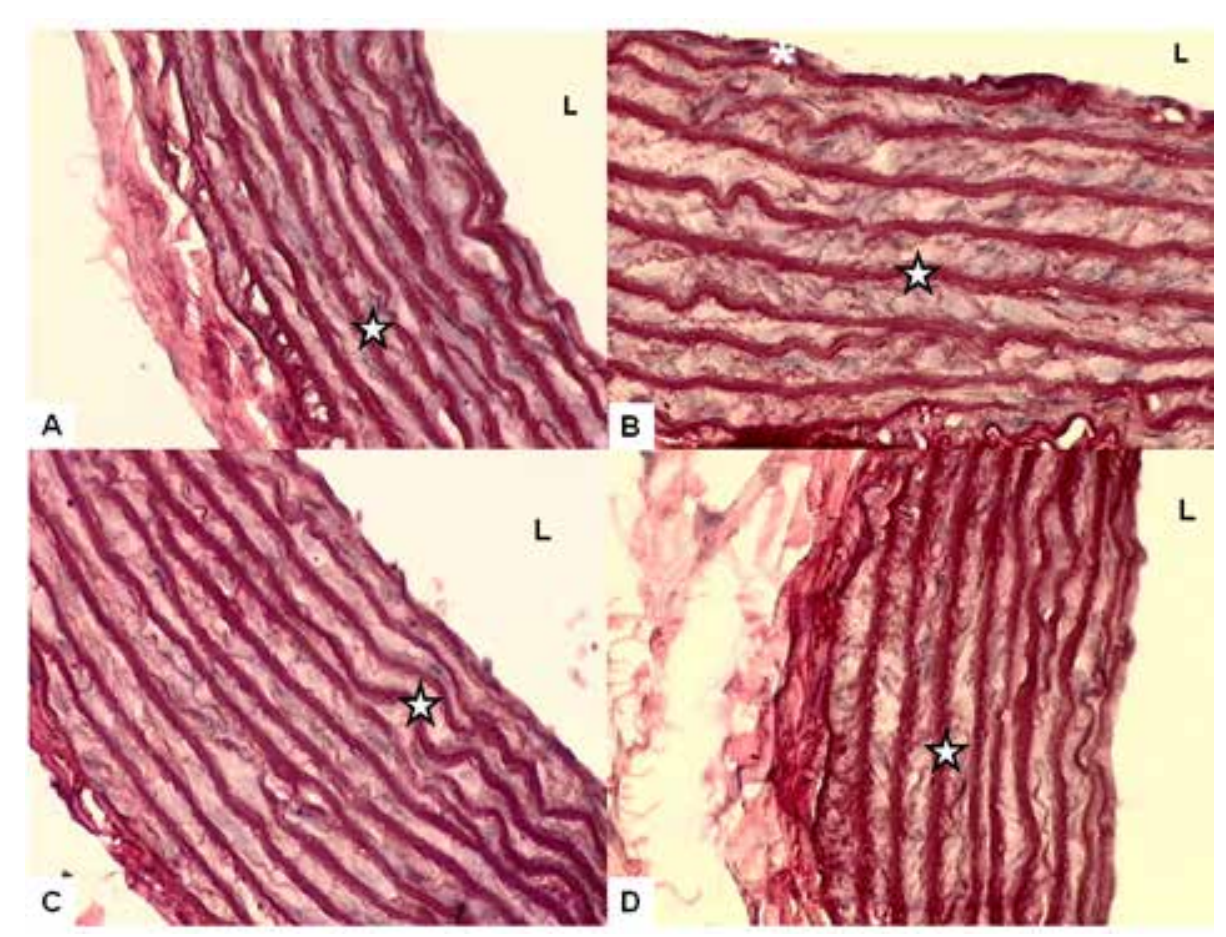

Figura 4. Fotomicrografia da aorta torácica dos grupos SCF (A), SCaF (B), TCF (C) e TCaF (D), evidenciando lâminas elásticas (estrela) na túnica média, duplicação da lâmina elástica interna (seta) com espessamento da íntima (asterisco) e a luz (L). Orceina, 640x 
Os resultados da espessura íntima-média $\mathrm{e}$ densidade de volume (Vv) do músculo liso e colágeno e a correspondente análise estatística estão apresentados na Tabela 1. A análise da espessura íntima-média mostrou diminuição na espessura da parede da aorta torácica no grupo TCaF, porém não foi significativo quando comparado com os demais grupos (Tabela 1). A análise da densidade de volume do músculo liso e do colágeno não mostrou diferenças significativas entre os quatro grupos (Tabela 1).

Tabela 1. Parâmetros morfométrico e de densidade de volume do colágeno e músculo liso da aorta torácica dos animais dos quatro grupos experimentais

\begin{tabular}{lccc}
\hline Grupos & $\begin{array}{c}\text { Vv Colágeno } \\
(\mu \mathbf{m})\end{array}$ & $\begin{array}{c}\text { Vv Músculo Liso } \\
(\mu \mathbf{m})\end{array}$ & $\begin{array}{c}\text { Espessura } \\
\text { intima-média } \\
(\mu \mathbf{m})\end{array}$ \\
\hline SCF & $0,39 \pm 0,03$ & $0,17 \pm 0,01$ & $104,6 \pm 5,9$ \\
SCaF & $0,39 \pm 0,03$ & $0,17 \pm 0,03$ & $92,37,4 \pm 5,42$ \\
TCF & $0,40 \pm 0,01$ & $0,19 \pm 0,02$ & $103,5 \pm 9,4$ \\
TCaf & $0,39 \pm 0,02$ & $0,17 \pm 0,03$ & $99,7 \pm 4,1$ \\
\hline
\end{tabular}

\section{DISCUSSÃO}

Este estudo investigou a associação entre a suplementação de fibras solúveis, a dieta de cafeteria e treinamento físico em aorta torácica de ratos Wistar. Este modelo de dieta vem sendo utilizado para induzir o aumento do peso corporal em animais em diferentes épocas de vida (DAMETO et al., 1994). A dieta de cafeteria, independente da utilização de fibras e do exercício físico, mostrou-se decisiva para o ganho de peso dos animais. No entanto, quando se associa uma dieta balanceada ao exercício físico e ao consumo de fibras alimentares o ganho de peso apresenta-se moderado.

Considerando que o ganho de peso está intimamente ligado ao perfil lipídico (OLIVEIRA et al., 2016), avaliamos esses parâmetros nos grupos estudados. Estudos epidemiológicos sugerem que a ingestão adequada de fibras reduz o risco de doenças cardiovasculares e doença cardíaca coronariana, principalmente através de uma redução nos níveis séricos de colesterol e lipoproteína de baixa densidade (LDL) sem afetar as concentrações de alta densidade de lipoproteína
(HDL) (GLORE et al., 1994; TRUSWEL, 1995; MIRA; GRAF; CANDIDO, 2009; SLAVIN, 2013). Assim, de acordo com os nossos resultados os valores do colesterol total e o LDL mantiveram-se inalterados, mostrando a capacidade das fibras solúveis em reduzir ou manter esses parâmetros.

No entanto, os efeitos das fibras solúveis nos lipídios plasmáticos não diferem nos níveis de triglicerídios e HDL (BROWN et al., 1999). Os grupos que receberam dieta de cafeteria, tanto sedentário quanto o treinado, apresentaram valores elevados de triglicerídios quando comparados ao controle, o que nos leva a concluir que a dieta rica em carboidratos e gorduras (dieta de cafeteria) mesmo associada ao uso de fibras alimentares e ao exercício físico torna-se primordial para o ganho de peso e alterações do perfil lipídico.

Quando avaliados os parâmetros histológicos relacionados à morfometria da aorta não foi notada influência da dieta e do treinamento físico. A suplementação associada ao exercício físico pode ter tido uma influência positiva na espessura da aorta, como pode ser observado no grupo $\mathrm{TCaF}$, que mesmo não tendo diferença significativa $(p=0,91)$, teve a menor média de espessura $(\mu \mathrm{m})$, mostrando assim que, mesmo com uma dieta hipercalórica, a suplementação associada ao exercício físico teve benefícios para a manutenção da espessura da aorta torácica, apresentando um vaso mais complacente.

O treinamento físico parece não causar alterações marcantes em animais, sem alterações do componente elástico, e podendo haver uma redução do componente colágeno (MATSUDA et al., 1993; FARIS, 1973; NIEDERHOFFER et al., 2000). O número de camadas de elastina varia dependendo do tamanho do animal e o padrão da média é muito variável em diferentes espécies, além de variar desde a íntima até a adventícia (SONG; ROACH, 1985). Neste estudo é claro que os animais com maior peso corporal também apresentaram maior desenvolvimento da espessura íntima-média, mas sem valores significativos.

Foi observado na análise da densidade de volume que o colágeno ocupa aproximadamente $40 \%$ do vaso e o músculo liso 17-19\%. O músculo liso ocupa aproximadamente $20 \%$ do peso da túnica média da aorta e o colágeno e elastina ocupam aproximadamente $60 \%$ 
(HARKNESS; HARKNESS; MCDONALD, 1967), dados semelhantes ao encontrado neste estudo. Esperavase com a dieta de cafeteria um aumento do colágeno e diminuição do músculo liso, ou seja, um vaso com característica de arteriosclerose, porém acredita-se que o exercício físico aliado à suplementação de fibras evitou esse processo.

\section{CONCLUSÃO}

$\mathrm{Na}$ presente pesquisa a fibra alimentar associada ao treinamento aeróbio e a uma dieta balanceada teve um resultado eficaz, não alterando a morfologia da aorta torácica.

A suplementação, a dieta de cafeteria e o exercício físico não influenciaram o índice de colesterol total e colesterol LDL dos quatro grupos em estudo, porém, a dieta de cafeteria, independente da utilização de fibras e do exercício físico, mostrou-se decisiva para o ganho de peso dos animais.

Este estudo possui limitações por não ter um grupo controle de suplementação de fibras, o que torna difícil a afirmação de que as fibras têm um efeito benéfico para preservação do vaso, melhora no perfil lipídico e peso corporal.

\section{REFERÊNCIAS}

BEHMER, O. A.; TOLOSA, E. M. C.; FREITAS NETO, A. G. Manual de técnicas para histologia normal e patológica. São Paulo: Edart, 1976. p. 121.

BERNAUD, F. S. R.; RODRIGUES, T. C. Fibra alimentar: ingestão adequada e efeitos sobre a saúde do metabolismo. Arq Bras Endocrinol Metab., v. 57, n. 6, p. 397-405, 2013.

BROWN, L.; ROSNER, B.; WILLETT, W. W.; SACKS, F. M. Cholesterol-lowering effects of dietary fiber: a metaanalysis. J Am Clin Nutr., v. 69, n. 1, p. 30-42, 1999.

COUTINHO, J. G.; GENTIL, P. C.; TORAL, N. A desnutrição e obesidade no Brasil: o enfrentamento com base na agenda única da nutrição. Cad. Saúde Pública, v. 24, n. 2, p. 332-340, 2008.

DAMETO, M. C.; RAYÓ, J. M.; ESTEBAN, S.; PRIETO, R. M.; TUR, J. A. Effect of cafeteria diet on alpha-MG intestinal absorption in rats. Comp Biochem physiol., v. 108, n. 2-3, p. 467-470, 1994.

DUFLONTH, D. L.; MORRIS, M.; MICHELINI, L. C. Modulation of exercise tachycardia by vasopressesin in the nucleus tractus solitari. Am J Physiology, v. 273, p. 1271-1282, 1997.

FARIS, A. W. The Effects of physical training upon the collagen content of the aorta of adult male white rats. J Sports Med., v. 13, p. 108-110, 1973.

GARCIA, R. W. D. Reflexos da globalização na cultura alimentar: considerações sobre as mudanças na alimentação urbana. Rev Nutr., v. 16, n. 4, p. 483-492, 2003

GLORE, S. R.; VAN TREECK, D.; KNEHANS, A. W.; GUILD, M. Soluble fiber and serum lipids: a literature review. $\mathbf{J}$ Am Diet Assoc., v. 94, n. 4, p. 425-36, 1994.

HARKNESS, M. L. R.; HARKNESS, R. D.; MCDONALD, D. A. The Collagen and elastin content of the arterial wall in the dog. Proc R Soc Lond B Biol Sci. v. 146, p. 541, 1967.

MANDICA, S.; MYERSA, J.; OLIVEIRA, R. B.; ABELLAA, J.; FROELICHERA, V. F. Characterizing differences in mortality at the low end of the fitness spectrum in individuals with cardiovascular disease. Eur J Cardiovasc Prev Rehabi. v. 17, n. 3, p. 289-295, 2010.

MATSUDA, M.; NOSAKA, T.; SATO, M.; OHSHIMA, M. Effects of physical exercise on the elasticity and elastic components of the rat aorta. Eur J Appl Physiol., v. 66, p. 112-126, 1993.

MATTOS, L. L.; MARTINS, I. S. Consumo de fibras alimentares em população adulta. Rev Saúde Pública, v. 34, n. 1, p. 50-55, 2000.

MIRA, G. S.; GRAF, H.; CÂNDIDO, L. M. B. Visão 
retrospectiva em fibras alimentares com ênfase em betaglucanas no tratamento do diabetes. Braz J Pharm Sci., v. 45 , n. 1, p. 11-20, 2009.

MONTEIRO, R. C. A.; RIETHER, P. T. A.; BURINI, R. C. Efeito de um programa misto de intervenção nutricional e exercício físico sobre a composição corporal e os hábitos alimentares de mulheres obesas em climatério. Rev Nutr., v. 17, n. 4, p. 479-489, 2004.

NEGRÃO, C. E.; MOREIRA, E. D.; SANTOS, M. C. L. M.; FARAH, V. M. A.; KRIEGER, E. M. Vagal function impairment after exercise training. J Apply Physiology, v. 72, p. 1749-1753, 1992.

NIEDERHOFFER, N.; KIEFFER, P.; DESPLANCHES, D.; LARTAUD-IDJOUADIENE, I.; SORNAY, M.; ATKINSON, J. Physical exercise, aortic blood pressure, and aortic wall elasticity and composition in rats. Hypertension, v. 35, p. 919- 924, 2000.

OLIVEIRA, P. M.; SILVA, F. A.; OLIVEIRA, R. M. S.; MENDES, L. L.; NETTO, M. P.; CÂNDIDO, A. P. C. Associação entre índice de massa de gordura e índice de massa livre de gordura e risco cardiovascular em adolescentes. Rev Paul Pediatr., p. 1-8, 2016.

PINHO, R. A.; ARAÚJO, M. C.; GHISI, G. L. M.; BENETTI, M. Doença Arterial Coronariana, Exercício Físico e Estresse Oxidativo. Arq Bras Cardiol., v. 94, n. 4, p. 549$555,2010$.

PROENCA, R. P. C. Alimentação e globalização: algumas reflexões. Cienc. Cult., v. 62, n. 4, p. 43-47, 2010.

RIQUE, A. B. R.; SOARES, E. A.; MEIRELLES, C. M. Nutrição e exercício na prevenção e controle das doenças cardiovasculares. Rev Bras Med Esporte, v. 8, n. 6, p. 244-254, 2002.

SLAVIN, J. Fiber and Prebiotics: Mechanisms and Health Benefits. Nutrients, p. 1417-1435, 2013.

SONG, S. H.; ROACH, M. R. A morphological comparison of aortic elastin from five species as seen with the scanning electron microscope. Acta Anat., n. 123, p. 45-50, 1985.
TRUSWELL, A. S. Dietary fibre and plasma lipids. Eur J Clin Nutr., v. 49, p. 105-9, 1995.

ZHANG, Z.; SHI, L.; PANG, W.; LIU, W.; LI, J.; WANG, H.; SHI, G. Dietary Fiber Intake Regulates Intestinal Microflora and Inhibits Ovalbumin-Induced Allergic Airway Inflammation in a Mouse Model. Plos One, v. 11, n. 2, p. 1-16, 2016.

Recebido: 10 de março de 2016

Revisado: 01 de abril de 2016

Aceito: 11 de abril de 2016 